\title{
Fourier transform infrared conformational investigation of type I collagen aged by in vitro induced dehydration and non-enzymatic glycation treatments
}

\author{
Maria Grazia Bridelli, Chiaramaria Stani, Roberta Bedotti \\ Department of Mathematical, Physical and Computer Sciences, University of Parma, Parma, Italy
}

\begin{abstract}
The two main ageing-inducing events in the collagenous tissues are the water loss and the formation of intermolecular crosslinks based on the reaction of collagen with matrix carbohydrates, following a mechanism known as non-enzymatic-glycation. With the aim to mimic the two deleterious processes for the protein structure, rat-tail collagen was submitted to hydration changes and allowed to interact with two sugars characterized by different reducing properties, D-glucose and D-ribose. Fourier transform infrared (FTIR) spectroscopy was employed to investigate the conformational changes induced in the protein by the two treatments by analyzing the subsequent spectra modifications. FTIR spectra monitored: i) the amplitude and position changes of the two characteristic absorption bands $\mathrm{OH}$ stretching and Amide I, in dependence on the humidity level: a significant hysteresis effect in
\end{abstract}

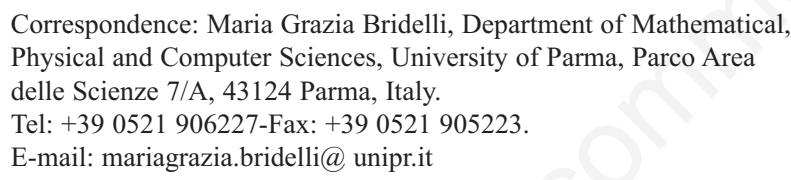

Key words: Collagen, Hydration, Non-enzymatic glycation, Ageing, FTIR spectroscopy.

Contributions: MGB conceived the experimental design, analyzed FTIR data and wrote the manuscript; CS collected and analyzed FTIR spectra and RB performed the preparation of collagen and collagensugar compounds. All authors helped with the discussion of the results and revised the submitted manuscript.

Conflict of interest: the authors declare no potential conflict of interest.

Conference presentation: part of this paper was presented at the $87^{\text {th }}$ SIBS National Congress, 2014 December 5-6, Turin, Italy.

Received for publication: 30 August 2016.

Accepted for publication: 19 December 2016.

(C)Copyright M.G. Bridelli et al., 2017

Licensee PAGEPress, Italy

Journal of Biological Research 2017; 90:6254

doi:10.4081/jbr.2017.6254

This article is distributed under the terms of the Creative Commons Attribution Noncommercial License (by-nc 4.0) which permits any noncommercial use, distribution, and reproduction in any medium, provided the original author(s) and source are credited. the $v(\mathrm{OH})$ band $\left(v \sim 3400 \mathrm{~cm}^{-1}\right)$ amplitude of the protein dehydrated and then rehydrated to the initial relative humidity $\left(a_{w}=0.92-\right.$ $0.06)$ may be related to the enhancement of the $\beta$-sheet fraction in the protein structure as revealed by the parallel modification in the Amide I band $\left(v \sim 1650 \mathrm{~cm}^{-1}\right)$; ii) the area of the carbohydrate double band peaking at $1080 \mathrm{~cm}^{-1}$ and $1031 \mathrm{~cm}^{-1}$, associated to the accumulation of the glycation products, depending on the sugar concentration and incubation time. The association of both sugars to collagen only minimally affects the protein secondary structure as revealed by Amide I band Gaussian analysis. The whole set of results suggests hints to hypothesize a self-assembly model for collagen molecules induced by ageing.

\section{Introduction}

Collagen is the most abundant protein in mammals and accounts for $25-30 \%$ of their protein content. It is the main fibrous component of skin, bone, tendon and cartilage, accounting in particular for three-quarters of the dry weight of skin, and representing the most prevalent component of the extracellular matrix. Collagen molecule is a rigid rod like structure able to resist stretching. It is composed of three polypeptide left-handed alpha chains coiled around each other to form a typical right handed rope like triple helical rod (approximatively $1.5 \mathrm{~nm}$ in diameter, $300 \mathrm{~nm}$ in length). The triple-helical sequences are comprised of Gly-X-Y repeats, $\mathrm{X}$ and $\mathrm{Y}$ being frequently proline and hydroxyproline, respectively. ${ }^{1,2}$ Stabilization of the triple helix is assured by a lot of structural conditions as the presence of glycine as every third residue, a high content of imino acids with a rigid cyclical structure (proline and hydroxyproline), and electrostatic interactions involving lysine and aspartate. ${ }^{3}$ However, a key role is undoubtedly played by water which provides inter-chain hydrogen bonds and water bridges, and coats triple helix by a cylinder-like of hydration. 4,5 Water critically regulates chain flexibility and assures the water mediated H-bonds favoring fiber recognition and alignment. Water depletion, caused by tissue maturation, exposition to UV light and/or some pathological processes, like diabetes, triggers non-enzymatic glycation reactions between the protein and the complex carbohydrates present in the matrix, leading to the formation of oxidation products, known as Advanced Glycation End-products (AGE). ${ }^{6}$ AGEs have the effect to modify the collagen fibers physical properties, inducing an increase in stiffness and fiber diameter resulting in a loss of solubility and flexibility and brittleness of the collagenous tissues. ${ }^{7}$

Several experimental techniques have been used in order to monitor and describe the changes in collagen structural properties as a consequence of ageing processes: high resolution X-ray dif- 
fraction, Scanning Electron Microscopy (SEM), Fluorimetry and Raman spectroscopy. ${ }^{8-11}$

Among the spectroscopic techniques, Fourier Transform InfraRed Spectroscopy (FTIR) is recognized as one of the most sensitive for studying biological macromolecules, being able to monitor changes in the structural features in dependence on the physicochemical and environmental conditions. ${ }^{12}$ In the present work, with the aim to investigate the role of both the reactions on the conformational properties of collagen, and how they influence one each other, the protein was submitted to hydration changes and allowed to interact with two sugars characterized by different reducing properties, D-glucose and D-ribose. ${ }^{13}$ Fourier Transform InfraRed (FTIR) spectroscopy was employed to investigate the conformational changes induced in the protein by the two treatments by analyzing the subsequent spectra modifications.

The typical IR spectrum of a protein is dominated by the Amide bands, due to the vibrations of the peptide bond. Amide I band, ranging between 1720 and $1610 \mathrm{~cm}^{-1}$, mainly due to contributions from the $\mathrm{C}=\mathrm{O}$ stretching vibration of the peptide backbone, is particularly sensitive to the secondary structure composition of the protein. To predict the protein conformation, Amide I band was decomposed into individual components, corresponding to the different types of secondary structure. ${ }^{14}$ Water structured around proteins displays strong absorption bands in the IR frequency range. ${ }^{15-17}$ A broad absorption band in the range $3700-3000 \mathrm{~cm}^{-1}$ due to the hydroxyl stretching vibrations $(v(\mathrm{OH}))$, and a relatively sharp but weaker one, caused by the bending mode $\delta(\mathrm{OH})$, peaking around $1640 \mathrm{~cm}^{-1}$. The $v(\mathrm{OH})$ profile and amplitude modification, in particular, as a consequence of the hydration fluctuations may be related to the reshaping of the water layer surrounding the bio-macromolecule and was correlated to the protein conformational changes.

Glycation compound formation was assessed by means of the identification in the IR spectrum of the protein/carbohydrate complex of an absorption band characteristic of the interaction between collagen and reducing sugars, such as Glucose and Ribose. ${ }^{11,17}$ Ribose and Glucose IR spectra are characterized by a lot of absorption bands largely superimposed to the main protein features, however the spectra of both the sugars are characterized by a huge absorption band in the $1150-950 \mathrm{~cm}^{-1}$ due to the glucid C-O-C, C$\mathrm{OH}, \mathrm{C}-\mathrm{O}$ and $\mathrm{C}-\mathrm{C}$ vibrations. As a consequence, glycated collagen displays significant spectral differences with respect to the native protein, in correspondence of the wave number range spanning such region (1150-950 $\left.\mathrm{cm}^{-1}\right)$. This band was assumed as a marker of the protein/carbohydrate interaction, as discussed in literature, ${ }^{11}$ and the modifications in the amplitude and area band were correlated to the glycation amount produced.

In the natural ageing processes dehydration and glycation may be considered as subsequent steps, where carbohydrates act as water substitutes, being rich in $\mathrm{OH}$ moieties, against the damages produced by moisture subtraction, but the structure rearrangements produced in collagen by the two reactions are not yet clarified. ${ }^{18,19}$ The purpose of the present preliminary study is to separately investigate the modifications produced in collagen structure by the two processes, dehydration and non-enzymatic glycation, in order to contribute to the elucidation and the spectroscopic characterization of both the ageing processes.

\section{Materials and Methods}

\section{Samples}

Collagen from rat tail (MW 102610) was purchased from
Sigma-Aldrich and used without further chemical purification. It was solubilized in acid acetic aqueous solution $(0.5 \mathrm{M})$.

Glucose and Ribose were purchased from Sigma-Aldrich.

The samples for FTIR measurements were thin films obtained by depositing a drop of protein solution on $\mathrm{CaF}_{2}$ platelets and allowing them to dry in air. The sample was vertically positioned in such a way as to allow for transmission measurements.

\section{Hydration-dehydration procedure}

Different hydration degrees of the samples were obtained by equilibrating them for two days directly in the inner ambient of a home-made dry box over suitable hydrating saturated salt solutions put in a vessel placed into the box and assembled together with the sample. In this way the relative humidity was made to vary in the activity range $a_{w}=0.06-0.92$, being $a_{w}=0.06$ provided by $\mathrm{NaOH}$, and $a_{w}=0.92$ by $\mathrm{KNO}_{3}$ saturated salt solutions. ${ }^{15}$

\section{Glycation reaction}

It was accomplished by incubating collagen under sterile conditions in Glucose and Ribose 5 and $50 \mathrm{mM}$ in phosphate buffer solutions $(0.15 \mathrm{mM}, \mathrm{pH} 7.4)$ at $37^{\circ} \mathrm{C}$. The reaction development was checked after 3,10 and 15 days by taking aliquots of solution and smearing them on the $\mathrm{CaF}_{2}$ platelets. The air dried films were equilibrated in the dry box at $a_{w}=0.92$ as above described.

\section{Fourier transform infrared spectroscopy}

Infrared spectra were collected with a Jasco 420 FTIR spectrophotometer operating at RT within the $4000-600 \mathrm{~cm}^{-1}$ range (128 scans, spectral resolution $2 \mathrm{~cm}^{-1}$ ).

Gaussian functions were used to fit Amide I and $v(\mathrm{OH})$ bands.

Amide I band $\left(1720-1610 \mathrm{~cm}^{-1}\right)$ was decomposed in subbands whose position and area were related respectively to the different types and to the amount of secondary structures. ${ }^{14}$ The sum of the areas of the peaks represents the total amount of secondary structure in the protein.

OH stretching band (wave number range 4000-3000 $\mathrm{cm}^{-1}$ ) was analyzed after subtraction of the spectrum of the same sample preheated to $80^{\circ} \mathrm{C}$ for $4 \mathrm{~h}$ assumed as dry sample. In this way any contribution to the band of Amide A (N-H stretching, $\left.v \sim 3300 \mathrm{~cm}^{-1}\right)$ and aliphatic $\mathrm{CH}_{2} / \mathrm{CH}_{3}$ stretching bands $\left(2960-2850 \mathrm{~cm}^{-1}\right)$, occurring in the same wave number range investigated, was systematically eliminated. The cleaned $\mathrm{OH}$ stretching band was decomposed into components whose frequencies were related to different $\mathrm{O}-\mathrm{H}$ bond lengths, i.e. to water molecule sets different in the vibrational energies as a consequence of the various interaction strengths, mutual and over the binding sites offered by the protein surface.

The area of the glycation band, the twin peak ranging between $1150-950 \mathrm{~cm}^{-1}$, was evaluated after baseline correction.

\section{Results and Discussion}

In Figure 1, FTIR spectra of pure collagen (a) and collagen glycated with ribose (50 mM, 15 days) (b) films, prepared at the highest attained water activity $\left(a_{w}=0.92\right)$ and recorded in the wave number range $4000-400 \mathrm{~cm}^{-1}$, are displayed and compared.

In the past 25 years infrared spectroscopy has produced a significant upgrade in structural studies of collagen: ${ }^{20-22}$ Although the IR spectra of the protein has already been shown and discussed in several papers in the past, ${ }^{21}$ the characteristic features are summa- 
rized here, for the sake of clarity. In the region between 3700 and $2600 \mathrm{~cm}^{-1} v(\mathrm{OH}), v(\mathrm{NH})$ and $v(\mathrm{CH})$ absorption bands characteristic for bound water $\left(\sim 3400 \mathrm{~cm}^{-1}\right)$, Amide $\mathrm{A}\left(3300 \mathrm{~cm}^{-1}\right)$ and $\mathrm{CH}_{3}, \mathrm{CH}_{2}$ groups of alkyl/acyl chains $\left(2950-2860 \mathrm{~cm}^{-1}\right)$. The skeletal functionality region, below $2000 \mathrm{~cm}^{-1}$, is dominated by the three main Amide bands, Amide I $\left(1650 \mathrm{~cm}^{-1}\right)$, Amide II $\left(1550 \mathrm{~cm}^{-1}\right)$ and Amide III (1340-1220 $\left.\mathrm{cm}^{-1}\right)$. The main IR-active groups are indicated in Figure 1 by arrows. The two spectra, a and b, are similar in the main features except for the low wave number range, between $1150-950 \mathrm{~cm}^{-1}$ where the typical twin band due to the carbohydrate moiety vibration modes shows, in the $b$ spectrum, a very larger amplitude with respect to the native collagen one (a spectrum).

\section{Hydration-dehydration treatments}

In order to study the role of water interacting with collagen, the FTIR spectrum was analyzed in the $\mathrm{OH}$ stretching region by changing the hydration content of the sample following the method described in the Experimental Section. The composition in Gaussian sub-bands of the $v(\mathrm{OH})$ feature can be correlated to the hydrogen bond network around the protein each component being assigned to different sets of $\mathrm{H}_{2} \mathrm{O}$ molecules each one related by $\mathrm{O}$ $\mathrm{H}$ bonds of comparable lengths. Figure 2A displays the absorption spectrum of the water bound to collagen prepared at $a_{w}=0.92$ (as described in Materials and Methods). The band was resolved, following the procedure of the second derivative analysis, into four Gaussian components $\left(\mathrm{R}^{2}=0.998\right)$ peaking at $3610,3513,3360$ and $3180 \mathrm{~cm}^{-1}$. They can be related to four classes of water molecules bound to the protein, different in vibrational energies and each one characterized by a single average $\mathrm{H}$-bond distance $(\mathrm{H} \bullet \bullet \mathrm{OH}$ length): $0.303,0.293,0.283,0.276 \mathrm{~nm}$, respectively. ${ }^{23}$

The component band at the highest wave number region is corresponding to $\mathrm{H}$-bond distances characteristic of vapor-like state. Similar features, detected in different biological macromolecules $^{16,24}$ were assigned to the non-H-bonded or weakly H-bonded

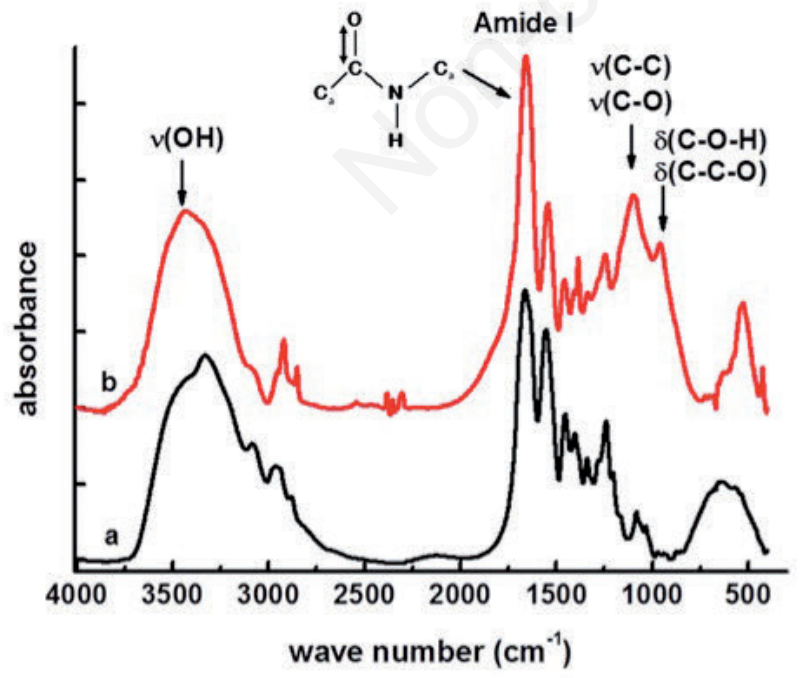

Figure 1. Fourier transform infrared spectra of (a) native collagen from rat tail tendon (SIGMA) and (b) collagen glycated for 15 days with glucose $(50 \mathrm{mM})$. Measurements were carried out in transmission geometry on dried films smeared on $\mathrm{CaF}_{2}$ slabs. To facilitate the comparison, the spectra were normalized with respect to the amount of protein in each sample.
$\mathrm{O}-\mathrm{H}$ groups. It is reasonable to suppose that in our sample, prepared at very low hydration levels, they could be originated by the most external water molecules sitting on the outer hydration layer coating the macromolecule. The inability for these molecules to establish active H-bonds with the surroundings, as in bulk water, accounts for the high mobility and the large vibrational energy comparable with those of the water molecules in the vapor state. They represent about the $5 \%$ of the total amount of water hydrating collagen at the relative humidity settled for the experiment, being the percentages calculated as the ratio $A_{i} / A_{\text {tot }}$ of the single component area $\left(A_{i}\right)$ with respect to the total band area $\left(A_{t o t}\right)$. The two component bands peaking at the intermediate wave numbers may be related to $\mathrm{H}_{2} \mathrm{O}$ molecules coordinated by two or three more or less distorted or strained $\mathrm{H}$ bonds. They may be identified with $\mathrm{H}_{2} \mathrm{O}$ molecules deeply located inside the protein helix, acting as water bridges within a single peptide chain and/or interconnecting the different $\alpha$-chains in the triple helix. They constitute the $19 \%$ and the $31 \%$, respectively, of the total water amount. The corresponding H-bond distances suggest they could represent the solvation molecules involving $\mathrm{C}=\mathrm{O}$ groups belonging to Glycine and Hydroxyproline moieties. ${ }^{4}$ The component at $3180 \mathrm{~cm}^{-1}$ may be attributed to water molecules located near to the protein surface and hydrogen bonded to polar and charged groups exposed to the macromolecule surface: they originate some ice-like tetrahedral structures more or less distorted in dependence on the orientation of the surface chemical groups. The corresponding average $\mathrm{H}$ bond length is in fact characteristic for arrangement of $\mathrm{H}_{2} \mathrm{O}$ molecules as in small solid water clusters, assuming the length $\mathrm{O} \cdots \mathrm{O}$ of hydrogen bonds in ice as $0.276 \mathrm{~nm} .{ }^{25}$ The broad profile of such a band testifies for the large distribution of water molecules in a wide con-
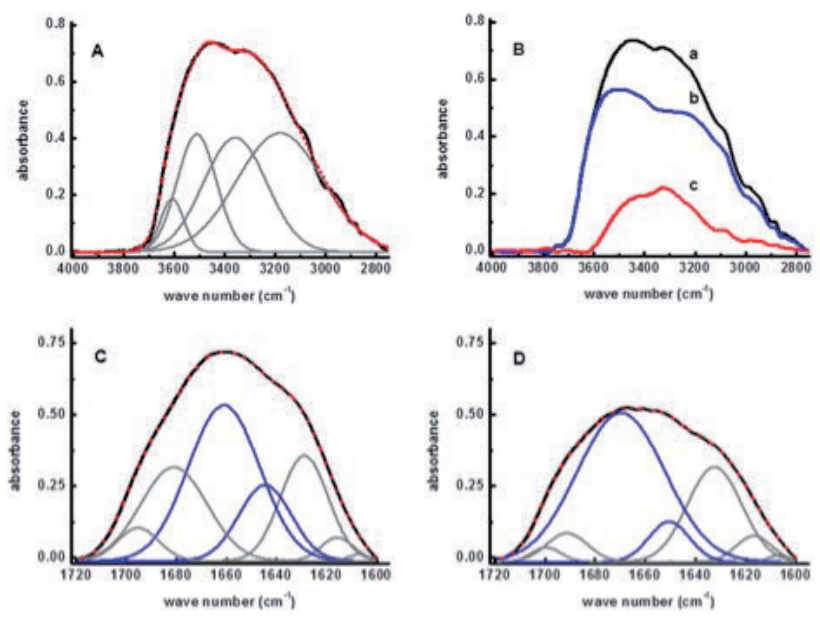

Figure 2. Gaussian deconvolution of $\mathrm{v}(\mathrm{OH})$ stretching band for native collagen hydrated at $a_{w}=0.92$, after subtraction of the dry sample spectrum (A). $v(\mathrm{OH})$ stretching band for collagen hydrated at $a_{w}=\mathbf{0 . 9 2}$ (line a) and after dehydration at $a_{w}=0.06$ and subsequent rehydration at $a_{w}=0.92$ (curve b). Curve c represents the difference spectrum (B). Amide I band deconvolution for native collagen at $a_{w}=0.92$ (C). Amide I band deconvolution for native collagen dehydrated at $\boldsymbol{a}_{w}=\mathbf{0 . 0 6}$ and thereafter rehydrated at $\boldsymbol{a}_{w}=\mathbf{0 . 9 2}$ (D). The sum of the fitted curves is shown by the thin grey lines, closely overlapping the experimental data trace, which is shown as a full heavy line. Blue lines in panels $\mathrm{C}$ and $\mathrm{D}$ indicate the component bands mainly modified by the dehydration-rehydration treatment. 
figuration variety, concurring to the feature. Such a sub-band represents the main hydration fraction at the settled humidity, corresponding to the $45 \%$ of the total amount of hydration water.

The dehydration treatment at $a_{w}=0.06$ followed by a subsequent rehydration up to the original relative humidity $\left(a_{w}=0.92\right)$ causes a pronounced hysteresis effect in the $v(\mathrm{OH})$ band amplitude recover and a remarkable modification of the profile (Figure $2 \mathrm{~B}$, curve b). In Figure 2B, curve c deserves particular note: it was obtained as the difference between curve a, corresponding to the hydrated sample, and curve b, representing the rehydrated one, after dehydration. It can be considered as the spectrum of water that can no longer be drawn back into the sample after the dehydration treatment. The broad band is peaking at $v \sim 3375 \mathrm{~cm}^{-1}$, therefore it is roughly filling the position of the third component of the original hydration band (a). Moreover it exhibits a shoulder on the lower wave number side $\left(v \sim 3100 \mathrm{~cm}^{-1}\right)$. This finding reveals that dehydration treatment gives rise in collagen to the loss of a considerable amount of $\mathrm{H}_{2} \mathrm{O}$ molecules involved in the internal water bridges and of a fraction of the molecules coating the polypeptide surface. These water molecules once desorbed, only partially can be re-adsorbed. It accounts for the $21 \%$ of the total water amount initially hydrating the sample.

Water deprivation has significant consequences on the macromolecule structure. They can be reflected by the changes in the low wave number region of the IR spectrum. The secondary structure analysis was carried out by analyzing the Amide I band in Gaussian components in the native sample and in the rehydrated one after dehydration. Qualitative and quantitative information about the conformational composition of the protein prepared in the different hydration states were obtained. The comparison of the spectra $\mathrm{C}$ and $\mathrm{D}$ in Figure 2 shows that the Amide I band amplitude of the rehydrated sample is reduced with respect to the freshly prepared one, as expected, due to the lacking of the water bending contribution in such wave number region $\left(\delta(\mathrm{OH}) \sim 1640 \mathrm{~cm}^{-1}\right)$. The curve fitting procedure allowed determining the secondary structure composition in the freshly prepared sample $\left(a_{w}=0.92\right.$, Figure 2C), as shown in Table 1: $\beta$-turn $\left(1695 \mathrm{~cm}^{-1}\right), \beta$-sheet $(1680$ $\left.\mathrm{cm}^{-1}\right), \alpha$-like helix $\left(1660 \mathrm{~cm}^{-1}\right)$, unordered structure $\left(1644 \mathrm{~cm}^{-1}\right)$, triple helix $\left(1628 \mathrm{~cm}^{-1}\right), \beta$-sheet $\left(1615 \mathrm{~cm}^{-1}\right)$, side chains $(1607$ $\mathrm{cm}^{-1}$ ). The multipeak decomposition of the band measured in the rehydrated sample (Figure 2D), revealed the modification occurred in the secondary structure. By normalizing the two peaks with respect to the maximum amplitude it was possible to compare the band shape. The procedure enabled the detection in the rehydrated sample of a band enlargement in the high wave number side. The most significant change in the conformational composition was in fact concerning the growth of a broad sub-band peaking at $v \sim$ $1670 \mathrm{~cm}^{-1}$ largely spanning the high wave number region of the Amide I band and the parallel decrease of the component bands at 1695 and $1660 \mathrm{~cm}^{-1}$. Such changes can be explained by assuming the formation of chemical bonds among collagen molecules laterally associating by forming structures side by side interacting. The formation of such structures may be envisaged as responsible for a relative increase of $\beta$ components with respect to the $\alpha$-helix contribution in the Amide I envelope. Therefore, as a consequence of the removal of water, the association of the collagen molecules takes place and once water is excluded, the assembled fibers are no able to re-adsorb all the water restituted during the rehydration phase, due to the lacking of available H-bonding sites. This arrangement is the main responsible for the decrease in the percentage amount of hydration water in the rehydrated sample and of the hysteresis effect measured in the $\mathrm{OH}$ stretching band.

\section{Non-enzymatic glycation reaction}

The Infrared marker for sugar and protein-sugar compounds is represented by a characteristic twin band in the fingerprint region, between $1150-950 \mathrm{~cm}^{-1}$, as shown by the $\mathrm{b}$ spectrum in Figure 1 . The band is peaking at $1032 \mathrm{~cm}^{-1}$ and at $1080 \mathrm{~cm}^{-1}$, the two peaks corresponding to C-O, C-C stretching vibrations and to C-O-H and $\mathrm{C}-\mathrm{C}-\mathrm{O}$ bending vibrations, respectively and can be used for the discrimination between native and glycated collagen by means of the amplitude increase displayed as a consequence of glycation reaction. ${ }^{18}$ No frequency shift was monitored but a change in amplitude as a function of the sugar concentration and the incubation time. This change was employed for kinetic monitoring of glycation process and for roughly estimating the glycation reaction development in dependence on the amount and type of sugars. The area under the Amide I band was assumed as indicative of collagen content and it was used for normalization of sugar peak areas used for measure the glycation product accumulation. Figure 3A shows the plot of normalized absorbance $v s$. sugar concentration as measured after 3, 10 and 15 days from the start of the reaction. The amount of glycation products increases with increasing sugar concentration, although no linear dependence was detected, as previously shown in literature. ${ }^{26}$ The extent of glycated collagen formation is markedly enhanced with the initial addition of sugar, indicating the high propensity to the interaction of protein with carbohydrates. Formation of complex is strongly sugar type dependent, the ribose being more potent in the reaction yield, in accordance with the difference in the reductive properties of the two sugars.

Figure 3B displays the temporal kinetic of the glycation reactions promoted by the two sugars at different concentrations ( 5 and $50 \mathrm{mM}$ ). The accumulation of the reaction products is linearly dependent on the time course of the reaction, monitored for 15 days, and the formation rate is higher for the reaction of collagen with ribose than with glucose.

Complementary investigations of the conformational modifications induced in the protein by the interaction with sugars was carried out once more by analyzing Amide I band in the spectra of collagen after incubation (15 days) with ribose and glucose (50 $\mathrm{mM}$ ). The results of the analysis are displayed in Table 1 where the percentage amount of each secondary structure motif detected in the native collagen is compared with the values obtained for collagen glycated with the two carbohydrates.

No significant difference in the conformational motifs were detected except for a light increase in the random coil amount and in the $\beta$-turn content respectively in collagen-glucose and in collagen-ribose products. The result demonstrates that the overall conformation of the collagen is not altered by sugar attachment and the recorded modifications, involving only negligible portions of the polypeptide may be interpreted as due more to local rearrangements of the structure, perhaps as a consequence of the incubation procedure, than to structural modifications induced by the glycation reaction.

\section{Conclusions}

In conclusion, FTIR spectroscopy is confirmed to be a technique particularly valuable for analyzing the conformational conversion of collagen as a consequence of dehydration and non-enzymatic glycation reaction with reducing sugars, the two main ageing inducing agents in the collagenous biological tissues such as skin, tendon and cartilage. The study was performed by correlating the 
Table 1. Secondary structure content in native collagen, collagen glycated with glucose, and collagen glycated with ribose, as evaluated from the Amide I band deconvolution.

\begin{tabular}{lcccc} 
Assignments & Band position $\left(\mathrm{cm}^{-1}\right)$ & Collagen $(\%)$ & Collagen+glucose* $\left.^{*} \%\right)$ & Coll+ribose \\
Lat. chains & 1607 & 1 & 1 & 1 \\
$\beta$ sheet & 1615 & 4 & 16 & 5 \\
\hline Tr. helix & 1628 & 17 & 27 & 16 \\
Unord & 1644 & 12 & 30 & 16 \\
\hline Helix/coil & 1660 & 38 & 18 & 28 \\
$\beta$ sheet & 1680 & 20 & 5 & 22 \\
\hline$\beta$ turn & 1695 & 8 & 12 \\
\hline
\end{tabular}

*Collagen glycated with glucose $(50 \mathrm{mM})$ for 15 days; ${ }^{\circ}$ collagen glycated with ribose $(50 \mathrm{mM})$ for 15 days.
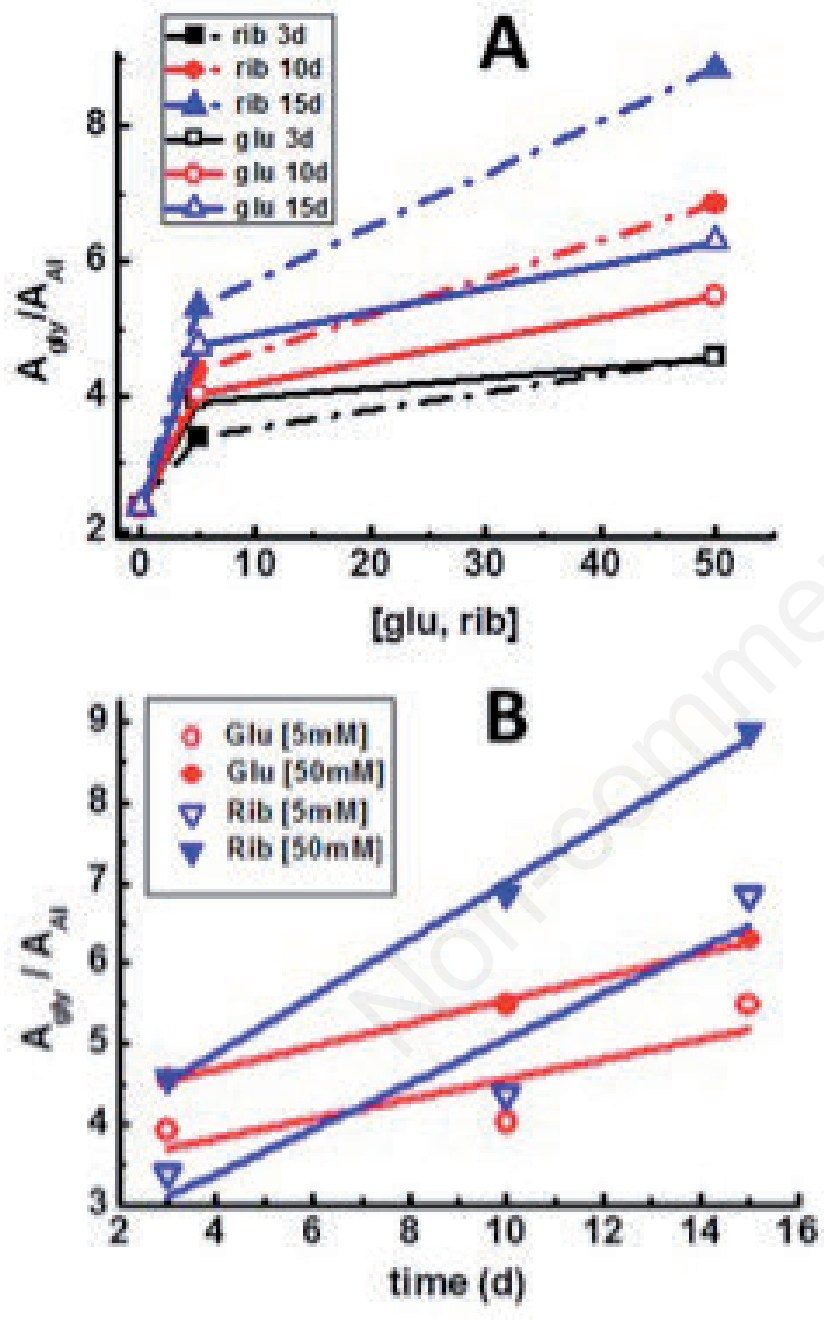

Figure 3. Glycation reaction product accumulation, as evaluated by the IR peak area in the wave number region $1150-950 \mathrm{~cm}^{-1}$ versus carbohydrate concentration. Full and empty symbols are related respectively to interaction with ribose and glucose: squares=incubation for 3 days; circles=incubation for 10 days; triangles=incubation for 15 days (A). Glycation reaction kinetics measured by the IR peak area in the wave number region 1150$950 \mathrm{~cm}^{-1}$, as a function of the incubation time measured in days. Circles: collagen-glucose; triangles: collagen-ribose (B). Empty symbols are corresponding to the lowest sugar concentration ( 5 $\mathrm{mM})$, the full symbols to the highest one $(50 \mathrm{mM})$ used in the reaction. The lines are a guide for the eyes. modifications in the Amide I band, reflecting the changes occurred in the secondary structure of the protein in dependence on the treatments to which it was submitted, with the two spectral features specifically monitoring the chemical groups directly involved in the reactions: $v(\mathrm{OH})$ band and the twin peak at 1080 and $1031 \mathrm{~cm}^{-1}$.

The main conclusions can be summarized as follows.

First, the results clearly stress the key role played by the hydration level on the FTIR spectrum and the extent by which water removal modifies the assembly properties of collagen. Molecular stability of the collagen structure is assured by four water classes different in the vibrational properties: two of these are related to inner helix $\mathrm{H}_{2} \mathrm{O}$ molecules, involving Gly and Hyp residues, and two to external water stratified in poly-molecular layers.

Second, dehydration-rehydration cycle induces lateral association of the fibers and shrinkage with a consequent squeezing out of a considerable fraction of external water unable to be re-adsorbed when the initial hydration level is restored. The effect is accompanied by an increase of $\beta$ sheet amount. On these bases a simple model can be hypothesized describing one of the phases of the most dramatic changes in collagenous tissues with age. It consists in the alignment and assembly of the collagen molecules towards fibers polymerization through intermolecular cross-linking water excluding.

Third, the interaction collagen-sugars can be monitored by analyzing the absorption band ranging between $1150-950 \mathrm{~cm}^{-1}$. Glycation reaction is dependent both on the concentration and the reducing properties of the sugar. In particular, the linear dependence of the glycation products amount on the incubation time may help to backtrack the early stage of the reaction in vivo and opens new research perspectives on the ageing of biological tissues providing improvements in many fields, for example in the dating of archaeological samples. ${ }^{27}$

Fourth, conformational analysis performed through Amide I deconvolution shows that collagen-sugar blends only minimally affect collagen secondary structure, confirming the protective role of sugars on protein conformational changes induced by deleterious effects such as tissue water loss. ${ }^{28}$

\section{References}

1. Perumal S, Antipova O, Orgel JPRO. Collagen fibril architecture, domain organization, and triple-helical conformation govern its proteolysis. Proc Natl Acad Sci USA 2008;105: 2824-29.

2. Shoulders MD, Raines RT. Collagen structure and stability. Annu Rev Biochem 2009;78:929-58. 
3. Kotch FW, Raines RTP. Self-assembly of synthetic collagen triple helices. Proc Natl Acad Sci USA 2006;103:3028-33.

4. Bella J, Brodsky B and Berman HM. Hydration structure of a collagen peptide. Structure 1995;3:893-906.

5. Kawahara K, Nishi Y, Nakamura S, et al. Effect of Hydration on the Stability of the Collagen-like Triple-Helical Structure of [4(R)-Hydroxyprolyl-4 $(R)$-hydroxyprolylglycine]. Biochemistry 2005;44:15812-22.

6. Bucala R, Cerami A. Advanced glycosylation: chemistry, biology and implications for diabetes and aging. Adv Pharmacol 1992;23:1-4.

7. Bailey AJ, Paul RG, Knott L. Mechanisms of maturation and ageing of collagen. Mechan Ageing Develop 1998;106:1-56.

8. Yamamoto S, Hashizume H, Hitomi J, et al. The subfibrillar arrangement of corneal and scleral collagen fibrils as revealed by scanning electron and atomic force microscopy. Arch Histol Cytol 2000;3:127-35.

9. Bai P, Phua K, Hardt T, et al. Glycation alters collagen fibril organization. Connect Tissue Res 1992;28:1-12.

10. Menter JM. Temperature dependence of collagen fluorescence. Photochem Photobiol Sci 2006;5:403-10.

11. Guilbert M, Said G, Happillon T, et al. Probing non-enzymatic glycation of type I collagen: A novel approach using Raman and infrared biophotonic methods. Biochim Biophys Acta 2013;1830:3525-31.

12. Mantsch HH, Chapman D. Infrared spectroscopy of biomolecules. New York, NY: John Wiley \& sons, inc.; 1996.

13. Bailey AJ, Sims TJ, Avery NC, Halligan EP. Non-enzymic glycation of fibrous collagen: reaction products of glucose and ribose. Biochem J 1995;305:385-90.

14. Barth A, Zscherp C. What vibrations tell us about proteins. Quart Rev Biophys 2002;35:369-430.

15. Bridelli MG, Capelletti R, Maraia F, et al. Initial hydration steps in lipase studied by means of water sorption isotherms, FTIR spectroscopy and thermally stimulated depolarization currents. J Phys D 2002;35:1039-48.

16. Bridelli MG, Crippa PR. Infrared and water sorption studies of the hydration structure and mechanism in natural and synthetic melanin. J Phys Chem B 2010;114:9381-90.

17. Ping ZH, Nguyen QT, Chen SM, et al. States of water in dif- ferent hydrophilic polymers-DSC and FTIR studies. Polymer 2011;42:8461-67.

18. Khajehpour M, Dashnau JL, Vanderkooi JM. Infrared spectroscopy used to evaluate glycosylation of proteins. Anal Biochem 2006;348:40-8.

19. Fessel G, Li Y, Diederich V, et al. Advanced glycation endproducts reduce collagen molecular sliding to affect collagen fibril damage mechanisms but not stiffness. PLoS One 2014;9:e110948.

20. Payne KJ, Veis A. Fourier Transform IR spectroscopy of collagen and gelatin solutions: deconvolution of the Amide I band for conformational studies. Biopolymers 1988;27:1749-60.

21. Petibois C, Gouspillou G, Wehbe K, et al. Analysis of type I and IV collagens by FT-IR spectroscopy and imaging for a molecular investigation of skeletal muscle connective tissue. Anal Bioanal Chem 2006;386:1961-6.

22. Noreen R, Moenner M, Hwu Y, Petibois, C. FTIR spectroimaging of collagens for characterization and grading of gliomas. Biotechnol Adv 2012;30:1432-46.

23. Nakamoto K, Margoshes M, Rundle RE. Stretching frequencies as a function of distances in hydrogen bonds. J Am Chem Soc 1955;77:6480-86.

24. Mallamace F, Chen S-H, Broccio M, et al. Role of the solvent in the dynamical transitions of proteins: The case of the lysozyme-water system. Proc Natl Acad Sci USA 2007;104:424-8.

25. Ben-Naim, A. Molecular theory of water and aqueous solutions. Part I: Understanding water. Singapore: World Scientific Publishing Co. Pte. Ltd.; 2010.

26. Roy R, Boskey A, Bonassar LJ. Processing of type I collagen gels using nonenzymatic glycation. J Biomed Mater Res A 2010;93:843-51.

27. Bridelli MG, Bedotti R, Stani C, et al. Studio delle modificazioni strutturali e conformazionali nel collagene fossile con tecniche di spettroscopia ottica. Ann Uni Ferrara Mus Sci Nat 2014;102:102-8.

28. Leslie SB, Israeli E, Lighthart B, et al. Trehalose and sucrose protect both membranes and proteins in intact bacteria during drying. Appl Environ Microb 1995;61:3592-7. 